\title{
Physical activity-friendly neighbourhood among older adults from a medium size urban setting in Southern Europe
}

\author{
Ana Isabel Ribeiro ${ }^{\mathrm{a}, \mathrm{b}, \mathrm{c}, *}$, Richard Mitchell ${ }^{\mathrm{d}, 1}$, Marília Sá Carvalho ${ }^{\mathrm{e}, 2}$, Maria de Fátima de Pina a,b,c,3 \\ a Departamento de Epidemiologia Clínica, Medicina Preditiva e Saúde Pública, Faculdade de Medicina, Universidade do Porto \\ b ISPUP - Instituto de Saúde Pública da Universidade do Porto \\ c INEB - Instituto de Engenharia Biomédica, Universidade do Porto \\ d Centre for Population Health Sciences, College of Medical, Veterinary and Life Sciences, University of Glasgow \\ e PROCC - Programa de Computação Científica, Fundação Oswaldo Cruz, Rio de Janeiro, Brazil
}

\section{A R T I C L E I N F O}

Available online 9 September 2013

\section{Keywords:}

Physical activity

Urban environment

Neighborhood

Portugal

Older adults

\section{A B S T R A C T}

Objective. In this cross-sectional study, we examined the relationship between socio-environmental characteristics of neighborhood of residence and the frequency of leisure-time physical activity (LTPA) among older adults from Porto (Portugal).

Method. Data from EpiPorto - a prospective adult cohort study from Porto (Portugal) - were used. Only adults aged $\geq 65$ at baseline $(1999-2003)$ were included $(n=580)$. We used a Geographic Information System to objectively measure the neighborhood characteristics and Generalized Additive Models to estimate their effect on participation in LTPA (none vs. some reported) and frequency of LTPA (min/day).

Results. $62 \%$ of the participants reported no LTPA. Active elderly spent on average 38 (women) and 67 (men) minutes per day exercising. Neighborhood characteristics were unrelated to whether older people exercised or not. However, among active individuals, distance to the nearest destination $(\beta=-0.154, p=0.016)$, in women, and distance to the nearest park, in men $(-0.030,0.050)$, were predictors of LTPA frequency.

Conclusion. There was almost no association between neighborhood characteristics and whether older adults engaged in LTPA or not, but among those that did engage, neighborhood characteristics were associated with increased frequency of LTPA. The promotion of well distributed destinations and parks might improve physical activity levels among the elderly.

(c) 2013 Elsevier Inc. All rights reserved.

\section{Introduction}

Along with a healthy diet and psychosocial well-being, physical activity (PA) is a major determinant for successful aging (Gremeaux et al., 2012). International guidelines among older adults recommend 150 min of moderate-vigorous PA per week (WHO, 2010) but even

\footnotetext{
* Corresponding author at: GeoEpidemiology Group, Instituto de Engenharia Biomédica INEB, Universidade do Porto, Rua do Campo Alegre, 823, 4150-180 Porto. Tel.: +351 226074900 ; fax: + 351226094567.

E-mail addresses: ana.isabel.ribeiro@ineb.up.pt (A.I. Ribeiro),

Richard.Mitchell@glasgow.ac.uk (R. Mitchell), carvalho@fiocruz.br (M.S. Carvalho),

fpina@med.up.pt (M.F. de Pina).

${ }^{1}$ Centre for Research on Environment, Society and Health, Institute of Health and Wellbeing, University of Glasgow, 1 Lilybank Gardens, Glasgow G12 8RZ. Tel.: + 441413 301663.

2 Fundação Oswaldo Cruz, Programa de Computação Científica, Vice Presidência de Ensino Informação e Comunicação, Av Brasil, 4365 - Antiga Residência Oficial, Manguinhos, 21045 900 - Rio de Janeiro, RJ - Brasil. Tel: +55 21 38361116; fax: +55 2122705141.

3 GeoEpidemiology Group, Departamento de Epidemiologia Clínica, Medicina Preditiva e Saúde Pública, Faculdade de Medicina, Universidade do Porto, Portugal, Alameda Prof. Hernâni Monteiro, 4200-319 Porto. Tel.: +351 225513652; fax: + 351225513653
}

light intensity PA is an important predictor of survival (Dogra and Stathokostas, 2012). Still, the majority of older people report sedentary lifestyles.

Several motivators and barriers may influence an older person's PA (Schutzer and Graves, 2004). While there has been a recent plethora of studies on the associations between PA and neighborhood characteristics, these focused predominantly on children, adolescents and working-age adults. But changes in functional and cognitive capacity, reductions in income and increasingly limited spaces for activity make seniors more vulnerable to the effects of local environment on health and related behaviors (Kawachi and Berkman, 2003).

Many environmental correlates of PA have been mooted. However, systematic reviews have found inconsistent results. The association between neighborhood attributes and PA is by no means proven (Cunningham and Michael, 2004; Koeneman et al., 2011; McCormack and Shiell, 2011). It is also likely that associations will vary by gender, and that gender differences may interact with age. Firstly, the older individuals are, the lower their mobility. Secondly, the use of space is highly dependent on social constructs. For instance, women appear to be more vulnerable to the neighborhood effects (Stafford et al., 2005). In addition, social environment seems particularly important in women, 
whereas physical environment predominantly affects men (Kavanagh et al., 2006).

However, several weaknesses can be pointed out in most of the studies exploring the relationships between environment and health-related behavior: 1) self-reported bias because of the use of subjective measures about neighborhood environment; 2) focus on large heterogeneous urban settings, disregarding smaller city neighborhood effects may be different; and 3) preference for multilevel models that contemplate neither the inter-area dependency (Chaix et al., 2005) nor the modifiable area unit problem, when results are influenced by the size/shape of the administrative divisions (Openshaw, 1984).

Our study addresses a current gap in the literature by examining, using a cross-sectional design, the relationship between socioenvironmental variables of neighborhood of residence and the frequency of leisure-time physical activity (LTPA) among older adults from Porto (Portugal).

\section{Methods}

Setting

Located in the northwest of Continental Portugal, Porto municipality had approximately 260,000 inhabitants in 2001(INE, 2001) distributed across $41.7 \mathrm{~km}^{2}$ It is near the Atlantic coast, along the Douro River estuary. Historically, Porto is an industrial and port city that competes with Lisbon in terms of economic power.

\section{Participants}

The EpiPorto Cohort encompasses a representative sample of adults aged 18-92 years living in Porto. Baseline evaluation was conducted from 1999 to 2003. Participants were recruited by random digit dialing using households as the sampling frame. After assessing the number and age of the residents of each household, a simple randomization was applied to select one eligible person among the permanent adult residents. In the case of a refusal, no replacement was permitted. The response rate was $70 \%$, resulting in a total of 2485 participants (Santos and Barros, 2003).

The local ethics committee approved the study protocol. The study was carried out according to the Helsinki Declaration and all participants completed the informed written consent form. A Geographical Information System (GIS) was used to georeference addresses. For the present study, only adults aged 65 or more at baseline were included $(\mathrm{N}=648)$. Three $(0.5 \%)$ were excluded because of bad quality of address data.

The Mini Mental State Examination (MMSE) was used to screen for cognitive impairment. Taking into account that MMSE is highly affected for educational levels and no consensual cut-off values exist (Schmand et al., 1995), individuals with scores below the 5th percentile were excluded according to educationadjusted cut-off values: $>14$ for $0-2$ years of schooling, $>21$ for $3-4$ years (the former levels of obligatory education in Portugal), $>23$ for $5-8$ years and $>25$ for more than 8 years of schooling. Accordingly, 35 participants (5.4\%) were excluded, reducing the sample to 610 individuals.

\section{Leisure-time physical activity assessment}

LTPA was evaluated using the EpiPorto Physical Activity Questionnaire to measure time and intensity of a wide range of activities, such as rest, transport, work, household and leisure, which includes walking and organized sports (Camões et al., 2010). Time spent (min/day) in LTPA was available for 580 individuals, our final sample. Participants were classified into two categories: inactive (no LTPA reported) and active (some LTPA even if insufficient).

Compared with the final sample, subjects with missing data on LTPA were older, less educated and had been more frequently employed in manual occupations $(p<0.001)$.

\section{Individual variable assessment}

Individual characteristics were obtained through structured questionnaires and variables established as important predictors of LTPA were included as confounders: age; marital status; educational attainment (number of schooling years); previous occupation (re-categorized into manual and non-manual workers); smoking status (smoker, occasional smoker, non-smoker and exsmoker); comorbidities (absence or presence of at least one of the following conditions - cardiovascular, respiratory, osteoarticular and musculoskeletal disorders, cancer, depression, cirrhosis and hypo/hyperthyroidism); residence in Porto for 20 years or more (yes/no); and body mass index (discretized according to the World Health Organization, WHO, cut-offs).

\section{Socio-environmental variable assessment}

Neighborhood characteristics included as independent variables in the statistical analysis were: socioeconomic status (SES) and population density of the census tract of residence, distances to the nearest park, sport space, destination and sea/riverside, density of street intersections and bus stops and average land gradient within $200 \mathrm{~m}$ (adopted as the walkable distance for older individuals, simply referred to below as neighborhood) around participant's residence. Since individual data refers to baseline evaluation (1999-2003) all neighborhood characteristics were collected for 2001.

Latent class analysis was used to model SES, from a set of socioeconomic indicators at census tract level, related to age and education/occupation of residents and housing characteristics obtained from Statistics Portugal (INE, 2001) (Alves et al. [results not published yet]). Three discrete classes of SES were identified for Porto - from the least to the most deprived. Census data were also used to compute population density.

Park boundaries and entrances were obtained from the city council digital maps. Public sports spaces were georeferenced using a Global Positioning System. Sport spaces were classified into those typically preferred by men ( $\mathrm{n}=$ 71 , football, volleyball, walking, tennis, table tennis, boccia, swimming, golf, athletics and cycling); or women $(n=25$, volleyball, walking, tennis, table tennis, boccia, swimming, golf, athletics and cycling). This classification was based on local Portuguese reports and international studies, which documented a clear mismatch between sports preferences in older women and men (Marin, 1988; Salvador et al., 2009; Warde, 2006).

Additionally, the position of common destinations was assessed (using exhaustive lists and Google Earth imagery): public medical care services (hospital and health centers), places of worship (churches and cemeteries), cultural infrastructure (libraries and museums), shopping centers and elementary schools (accessed for leaving and picking up grandchildren).

Distances to parks, sport spaces, destinations and sea/riverside were calculated by the shortest street route from residence to the nearest feature.

Bus stop locations were obtained from STCP (Society for Collective Transports of Porto) and average land gradient (\%) was computed using a digital elevation model ( scale $=1: 25,000$ ) from the Army Geographical Institute.

The location of the participant's residence and socio-environmental attributes are depicted in Fig. 1.

\section{Statistical analysis}

Descriptive statistics were computed for all variables, by sex and degree of participation in LTPA. Mann-Whitney $U$ and Chi-square tests were employed to compare distributions and proportions - differences were confirmed at a significance value $\leq 0.05$.

For data modeling, LTPA was used as a dependent variable and individual and neighborhood characteristics as covariates. Firstly, the null hypothesis that LTPA doesn't depend on the spatial location of an individual's residence was tested. Secondly, univariate analysis was conducted and all covariates with $p$-values $\leq 0.10$ were included in the initial multivariate model. Then, each covariate was removed step by step until the final adjusted model was attained, eliminating consecutively those with the highest $p$-values. The final model included only covariates with $p$-values $\leq 0.05$ and a function (thin plate spline) applied on the coordinates of each participant's residence. The presence of interactions was evaluated by including interaction terms between gender/ marital status and area variables.

Two phases of models were built to test the hypotheses that 1 ) neighborhood characteristics were related to participation in LTPA and 2) neighborhood characteristics affect the time spent on LTPA among already-active persons. The first model (Eq. (1)) included the whole sample and assessed LTPA as a dichotomous variable (active/inactive). The second (Eq. (2)) contained only active individuals and assessed LTPA as a continuous variable ( $\mathrm{min} /$ day). Given its skewed distribution, the variable LTPA was log-transformed. The equations are presented below:

$\ln \left(y_{i}\right)=\beta_{0}+\sum \beta_{k} x_{i k}+f\left(\right.$ north $_{i}$, east $\left._{i}\right)+e_{i}$

$z_{i}=\beta_{0}+\sum \beta_{k} x_{i k}+f\left(\right.$ north $_{i}$, east $\left._{i}\right)+e_{i}$ 


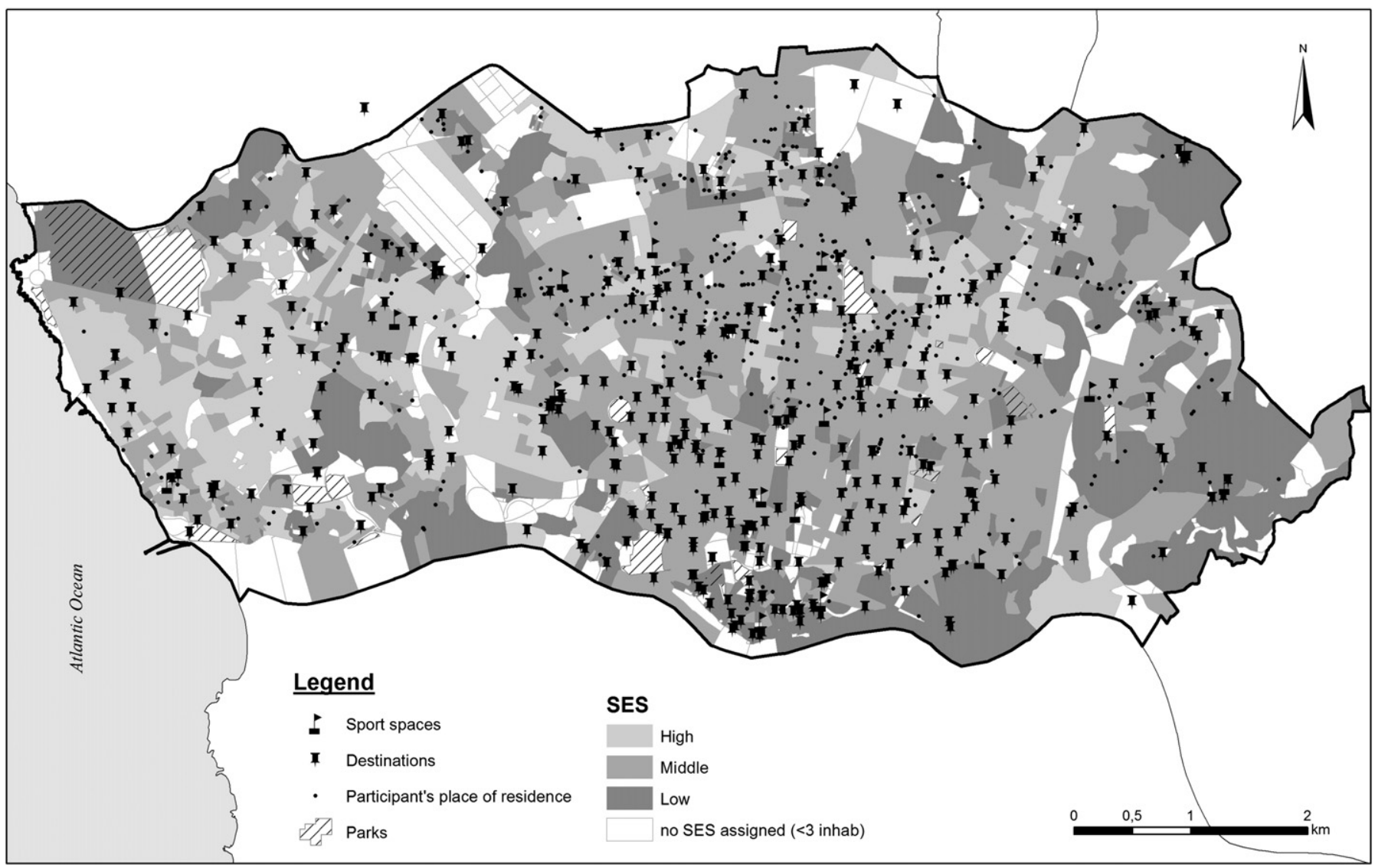

Fig. 1. Spatial distribution of the participant's residences and built and socio-environmental features (Porto, 1999-2003).

where $y_{i}$ is any LTPA, $z_{i}$ is the logarithm of the time spent on LTPA, $\beta$ s are the coefficients of the model, $x_{i k}$ are the explanatory variables, $f\left(\right.$ north $_{\mathrm{i}}$, east $\left.\mathrm{i}_{\mathrm{i}}\right)$ is a smooth function of the coordinates and $e_{i}$ are the residuals.

Additionally, the shape of significant relationships was estimated using thin plate splines and graphically represented. Due to the presence of interactions between sex and some neighborhood characteristics, sex-stratified models were built.

All analyses were conducted in R using the packages 'mgcv' (Wood, 2009), 'spatstat' (Baddeley and Turner, 2013) and 'spdep' (Bivand, 2013). ArcMap was used to georeference addresses, assess neighborhood characteristics, calculate routes and map results.

\section{Results}

\section{Descriptive statistics}

Table 1 shows the demographic and neighborhood socioenvironmental characteristics of the study sample.

From the total sample of 580 participants ( $57.9 \%$ women), $62.4 \%$ reported no LTPA. The mean age was around 72 years old for both sexes. Most of the participants were married; among women, there were a large proportion (39.3\%) of widows. Contrary to men, women were more likely to have been previously employed in manual professions. The mean number of schooling years was 5 and 7 years, respectively for women and men $(p<0.001)$. Overweight was the most common BMI condition but gender differences were found $(p<0.001)$. While there were very few female smokers, only $34.8 \%$ of the men reported they had never smoked $(p<0.001)$. Around $60 \%$ of the women and $32 \%$ of the men reported at least one illness $(p<0.001)$.

Mean distance to the closest park, sport space and destination was below $1 \mathrm{~km}$, reflecting equal opportunities to Porto residents. The average street intersection density was 3 nodes per hectare and participants had on average 3 bus stops around their residence. The average distance to the coast or riverside was more than $3 \mathrm{~km}$. The majority of participants lived in medium SES neighborhoods. Distance to suitable sport spaces was greater $(p<0.001)$ for women: around $800 \mathrm{~m}$ to the nearest feature. With the exception of neighborhood population density - lower among the active - no other neighborhood or individual variable differed significantly between active and inactive participants.

Among active individuals, the mean LTPA was 38.4 (women) and 66.9 (men) minutes per day. Only $11 \%$ of the females and $8 \%$ of the males fulfilled the WHO recommendations for PA (150 min per week of moderate-to-vigorous intensity).

Missing values were rare - a maximum of 13 cases for BMI.

\section{Generalized Additive Models}

There was no spatial autocorrelation in the distribution of participation in LTPA (either active/inactive or min/day). Thus, the spatial smoothing term was excluded from the models.

Results from the first phase of modeling (logistic regression with all participants, predicting any LTPA) showed that neighborhood characteristics - apart from population density in men $(\mathrm{OR}=0.995, p=0.013)$ had a limited effect on LTPA among older people. The model had a poor explanatory capability: $9.4 \%$ (men) and $6.0 \%$ (women) of the variability of the response (results not shown).

Table 2 shows the unadjusted and adjusted results of the second phase of modeling (already-active persons). Unadjusted analysis (Model 1) revealed a significant negative influence of neighborhood SES in LTPA in men and a positive effect among women. Distance to the nearest destination was negatively associated with women's LTPA.

After adjustment for potential confounders (Model 2), distance to destinations remained significant with women, with a negative effect on LTPA $(\beta=-0.1536, p=0.016)$. For every increase of $100 \mathrm{~m}$ in the distance to the nearest destination, the time spent in LTPA reduced 
Table 1

Characteristics of the participants (Porto, 1999-2003) according to participation in LTPA (inactive or active)

\begin{tabular}{|c|c|c|c|c|c|c|}
\hline & \multicolumn{2}{|l|}{ Total $(\mathrm{N}=580)$} & \multicolumn{2}{|l|}{ Inactive $(\mathrm{N}=362$ ) } & \multicolumn{2}{|l|}{ Active $(\mathrm{N}=218)$} \\
\hline & Women $(\mathrm{N}=336)$ & Men $(\mathrm{N}=244)$ & Women $(\mathrm{N}=232)$ & Men $(N=130)$ & Women $(\mathrm{N}=104)$ & Men $(\mathrm{N}=114)$ \\
\hline & Mean (SD) $)^{\mathrm{a}}$ or \% & Mean (SD) or \% & Mean (SD) or \% & Mean (SD) or \% & Mean (SD) or \% & Mean (SD) or \% \\
\hline Age (years) & $71.8(5.2)$ & $2.0(5.4)$ & $71.9(5.4)$ & $71.8(5.7)$ & $71.5(4.9)$ & $72.3(4.9)$ \\
\hline \multicolumn{7}{|l|}{ Marital status* } \\
\hline Married & 44.6 & 86.5 & 44.0 & 87.7 & 46.2 & 85.1 \\
\hline Single & 9.8 & 0.4 & 9.5 & 0.8 & 10.6 & 0.0 \\
\hline Widowed & 39.3 & 9.8 & 40.9 & 8.5 & 35.6 & 11.4 \\
\hline Divorced/separated & 6.3 & 3.3 & 5.6 & 3.1 & 7.7 & 3.5 \\
\hline Education attainment (no. years) ${ }^{*}$ & $5.0(3.9)$ & $6.8(4.2)$ & $4.5(3.5)$ & $7.1(4.6)$ & $6.2(4.5)$ & $6.6(3.7)$ \\
\hline \multicolumn{7}{|l|}{ Type of occupation ${ }^{*}$} \\
\hline Non-manual & 29.8 & 61.7 & 27.2 & 62.8 & 35.6 & 60.5 \\
\hline Manual & 70.2 & 38.3 & 72.8 & 37.2 & 64.4 & 39.5 \\
\hline Residence in Porto (>20 years) & 82.1 & 81.1 & 80.6 & 79.2 & 85.6 & 83.3 \\
\hline \multicolumn{7}{|l|}{ Comorbidities* } \\
\hline No & 39.9 & 67.6 & 42.2 & 72.3 & 34.6 & 62.3 \\
\hline Yes & 60.1 & 32.4 & 57.8 & 27.7 & 65.4 & 37.7 \\
\hline \multicolumn{7}{|l|}{ Body mass index ${ }^{*}$} \\
\hline Underweight $(<18.5)$ & 0.3 & 1.2 & 0.5 & 0.8 & 0.0 & 1.8 \\
\hline Normal (18.5-24.9) & 17.5 & 33.6 & 15.8 & 31.0 & 21.2 & 36.6 \\
\hline Overweight (25.0-29.9) & 47.5 & 46.5 & 46.4 & 48.1 & 50.0 & 44.6 \\
\hline Obese $(\geq 30.0)$ & 34.7 & 18.7 & 37.4 & 20.2 & 28.8 & 17.0 \\
\hline \multicolumn{7}{|l|}{ Smoking habits* } \\
\hline Smoker & 1.2 & 14.8 & 0.9 & 15.4 & 1.9 & 14.0 \\
\hline Occasional smoker & 0.3 & 0.8 & 0.4 & 0.8 & 0.0 & 0.9 \\
\hline Non-smoker & 93.7 & 34.8 & 93.9 & 33.8 & 93.3 & 36.0 \\
\hline Ex-smoker & 4.8 & 49.6 & 4.8 & 50.0 & 4.8 & 49.1 \\
\hline $\operatorname{LTPA}^{\mathrm{c}}(\mathrm{min} / \text { day })^{*}$ & $11.9(29.2)$ & $1.3(53.0)$ & 0 & 0 & $38.4(41.8)$ & $66.9(60.3)$ \\
\hline Met WHO recommendations (150 MVPA min/week) ${ }^{\mathrm{d}}$ & 3.3 & 33.7 & 0.0 & 0.0 & 10.6 & 7.9 \\
\hline Distance to the nearest park ( $\mathrm{hm})$ & $8.3(5.7)$ & $88.6(5.6)$ & $8.1(5.4)$ & $8.7(5.3)$ & $8.6(6.3)$ & $8.4(5.9)$ \\
\hline Distance to the nearest suitable sport space $(\mathrm{hm})^{*}$ & $8.1(4.2)$ & $55.0(2.9)$ & $8.0(4.1)$ & $5.2(3.0)$ & $8.2(4.6)$ & $4.9(2.9)$ \\
\hline Distance to the nearest destination (hm) & $2.3(1.5)$ & $23.3(1.4)$ & $2.3(1.5)$ & $2.4(1.5)$ & $2.2(1.4)$ & $2.3(1.4)$ \\
\hline Distance to the sea/riverside (hm) & $30.5(11.3)$ & $330.8(10.2)$ & $30.9(11.0)$ & $31.1(10.0)$ & $29.6(11.9)$ & $30.4(10.4)$ \\
\hline Population density (inhabit/ha) ${ }^{\#}$ & $121.9(73.6)$ & $1123.6(78.9)$ & $124.7(76.1)$ & $134.1(81.2)$ & $115.8(67.6)$ & $111.6(74.8)$ \\
\hline Intersection density (nodes/ha) ${ }^{\mathrm{e}}$ & $3.4(1.8)$ & $33.3(1.9)$ & $3.4(1.7)$ & $3.4(1.9)$ & $3.4(2.0)$ & $3.2(1.8)$ \\
\hline Bus stops ${ }^{\mathrm{e}}$ & $3.3(1.9)$ & $33.2(1.9)$ & $3.4(2.0)$ & $3.2(1.9)$ & $3.1(1.8)$ & $3.3(1.8)$ \\
\hline Land gradient $(\%)^{\mathrm{e}}$ & $2.6(1.5)$ & $22.5(1.4)$ & $2.6(1.5)$ & $2.4(1.3)$ & $2.5(1.6)$ & $2.5(1.5)$ \\
\hline \multicolumn{7}{|l|}{ Neighborhood SES $S^{b}$} \\
\hline 1 - least deprived & 18.5 & 221.3 & 16.8 & 24.6 & 22.1 & 17.5 \\
\hline 2 - medium deprived & 64.9 & 660.7 & 65.5 & 60.0 & 63.5 & 61.4 \\
\hline 3 - most deprived & 16.7 & 118.0 & 17.7 & 15.4 & 14.1 & 21.1 \\
\hline
\end{tabular}

a $\mathrm{SD}=$ standard deviation.

b $\mathrm{SES}=$ socioeconomic status.

c LTPA = leisure-time physical activity.

${ }^{d}$ WHO $=$ World Health Organization, MVPA $=$ moderate-to-vigorous physical activity.

e Within 200 meter circular buffer.

$* p \leq 0.05$ comparing men and women.

\# $p \leq 0.05$ comparing active and inactive.

by $14.2 \%\left(\left(1-e^{\beta 1}\right) \times 100\right)$. Among men, it was distance to parks that showed a slightly detrimental effect on LTPA $(\beta=-0.0298, p=$ 0.050 ): for every 100 meter increase in distance, LTPA decreased by $2.9 \%$. No other variables were significantly associated. Overall, the models accounted for $12.6 \%$ (women) and $14.5 \%$ (men) of the variability in LTPA.

We examined the functional form of the associations shown in Table 2 and detected a linear dose-response relationship between LTPA and distance to destinations among women (Fig. 2). In men, however, the relationship was curvilinear, less clear and only significant up to $500 \mathrm{~m}$ (Fig. 2).

\section{Discussion}

We examined the relationship between several objectively measured neighborhood characteristics and the frequency of LTPA in older people from Porto. Neighborhood characteristics were unrelated to whether they were physically active or not. When analysis was restricted to those active in some way, only distance to the nearest destination, in women, and distance to the nearest park, in men, were (negatively) associated with LTPA.

The direction of associations between LTPA and environmental characteristics was as expected and in line with findings from other studies. Proximity to shops, schools, cultural sites and places of social interaction may encourage older adults to perform PA, and has been associated with different types of PA (Inoue et al., 2011; Michael et al., 2006; Nagel et al., 2008; Nathan et al., 2012; Siu et al., 2012; Van Cauwenberg et al., 2012). We observed this association only for women and this, too, is echoed in the literature (Inoue et al., 2011; King et al., 2005; Van Dyck et al., 2012). Older women, especially in traditional societies such as Portugal, are usually responsible for domestic tasks - shopping, leaving grandchildren at school - and involved in 
Association between daily minutes spent in leisure-time physical activity (log-transformed) and neighborhood characteristics, stratified by sex (Porto, 1999-2003).

\begin{tabular}{|c|c|c|c|c|c|c|c|c|}
\hline & \multicolumn{4}{|l|}{ Model $1^{\mathrm{a}}$} & \multicolumn{4}{|l|}{ Model $2^{\mathrm{b}}$} \\
\hline & \multicolumn{2}{|l|}{ Women } & \multicolumn{2}{|l|}{ Men } & \multicolumn{2}{|l|}{ Women } & \multicolumn{2}{|l|}{ Men } \\
\hline & Coefficient & $p$-Value & Coefficient & $p$-Value & Coefficient & $p$-Value & Coefficient & $p$-Value \\
\hline Distance to the nearest park (m) & -0.0265 & 0.051 & -0.0230 & 0.124 & - & - & -0.0298 & 0.050 \\
\hline Distance to the nearest sport space (m) & 0.0055 & 0.770 & 0.0219 & 0.477 & - & - & - & - \\
\hline Distance to nearest destination (m) & -0.1675 & 0.004 & 0.0459 & 0.468 & -0.1536 & 0.016 & - & - \\
\hline Distance to the sea/riverside (m) & -0.0051 & 0.482 & -0.0042 & 0.626 & - & - & - & - \\
\hline Population density (inhabit/ha) ${ }^{\mathrm{c}}$ & -0.0015 & 0.231 & 0.0003 & 0.788 & - & - & - & - \\
\hline Street intersection density (nodes/ha) ${ }^{\mathrm{c}}$ & -0.0329 & 0.455 & 0.0098 & 0.842 & - & - & - & - \\
\hline Bus stops ${ }^{c}$ & -0.0233 & 0.625 & 0.0319 & 0.509 & - & - & - & - \\
\hline Land gradient $(\%)^{\mathrm{c}}$ & -0.0029 & 0.958 & -0.0044 & 0.940 & - & - & - & - \\
\hline Neighborhood SES ${ }^{\mathrm{d}}$ & & & & & - & - & - & - \\
\hline 1 - least deprived & Ref & & Ref & & & & & \\
\hline 2 - medium deprived & -0.2436 & 0.245 & 0.4390 & 0.066 & & & & \\
\hline $3-$ most deprived & -0.6442 & 0.026 & 0.2161 & 0.445 & & & & \\
\hline
\end{tabular}

a Univariate analysis.

b Multivariate analysis, adjusted for age, educational attainment, marital status, type of occupation, comorbidities, BMI and smoking habits.

c Within 200 meter circular buffer.

d $\mathrm{SES}=$ socioeconomic status.

church activities. It is thus reasonable to expect that the vicinity of those destinations might increase PA.

The association between proximity to parks and LTPA among men has been explored in other studies (Hanibuchi et al., 2011; Li et al., 2005; Siu et al., 2012). In our work, the distance from parks was negatively associated with PA among men, but the strength of this relationship was weak, indicating its contribution might be minor. Indeed, the literature finds the association for men to be relatively inconsistent and perhaps dependent on park attributes, and that associations for women are more usually absent (Hall and McAuley, 2010; Inoue et al., 2011; Nagel et al., 2008; Strath et al., 2012; Van Cauwenberg et al., 2012). Several studies have noted that the use of green space differs by gender: women tend to visit public parks less frequently and avoid them if they are unsafe and/or neglected. Men, in contrast, report fewer concerns about safety and tend to visit them often (Foster et al., 2004; O'Brien, 2005; Richardson and Mitchell, 2010). We could not assess the effect of public safety or quality of parks on LTPA, due to absence of data. However, since Porto is known to be a rather secure city, public safety might not be a key variable.

The absence of a relationship between LTPA and distance to sport facilities diverges from other studies (Cerin et al., 2012; Giehl et al., 2012;
Li et al., 2005). The lack of association in our study might result from a preference among Porto's older population for walking as PA, as in other settings (Cohen-Mansfield et al., 2004), thus not requiring exercise facilities. However, our data did not allow us to distinguish walking from other forms of PA.

No association between LTPA and street connectivity and density of bus stops was observed, which contrasts with studies where older people living in highly connected areas have been found to be more active (Hall and McAuley, 2010; Li et al., 2008). However, many other studies found no association (Hanibuchi et al., 2011; Nagel et al., 2008; Strath et al., 2012).

Land gradient and distance to sea or riverside had no effect on LTPA, but population density seemed to slightly inhibit PA. To our knowledge only one study has found such a negative relationship before (Tsunoda et al., 2012), while others found no clear association (Gomez et al., 2010; Hanibuchi et al., 2011). Unmeasured characteristics associated with dense urban areas - noise, unsightliness, traffic and crime - could explain our findings. These features have been examined elsewhere, and have been found to have a negative association with PA (Gomez et al., 2010; Kremers et al., 2012; Li et al., 2005; Nagel et al., 2008; Strath et al., 2012; Van Cauwenberg et al., 2012).
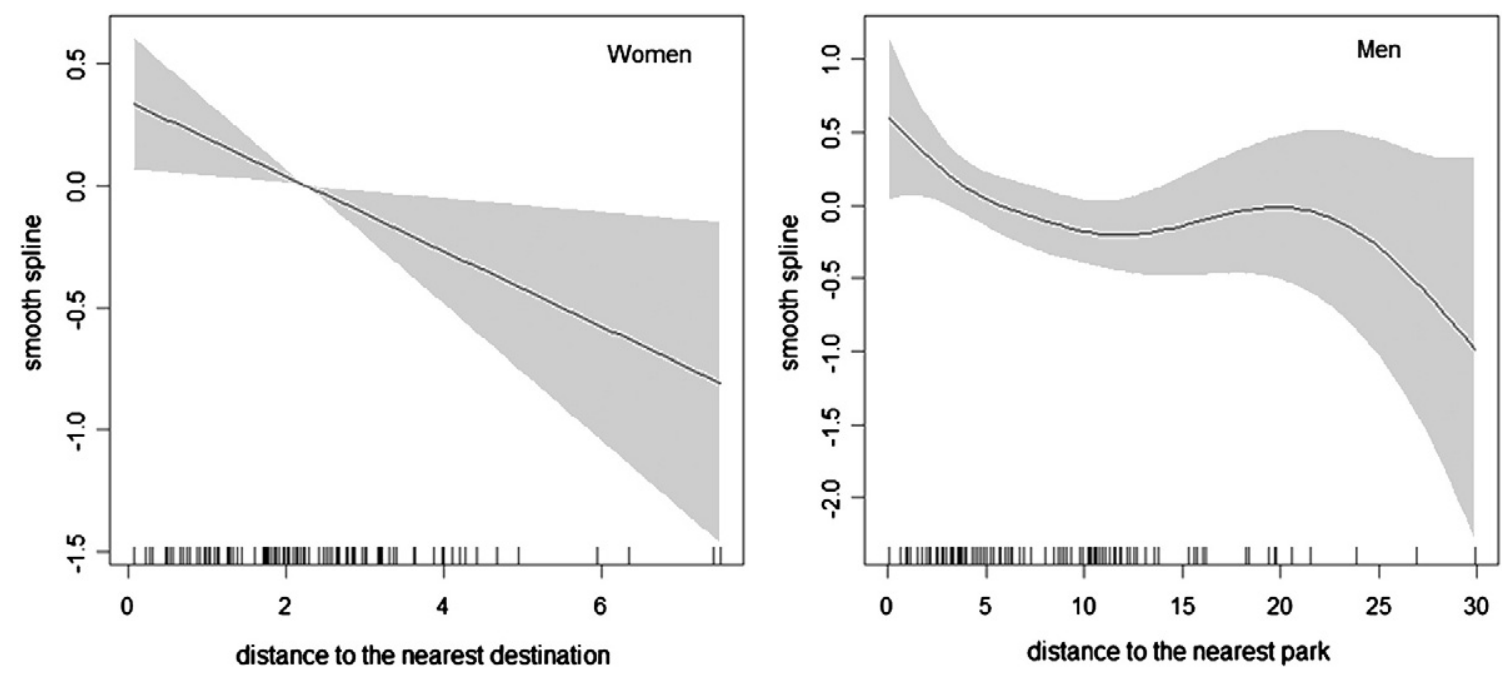

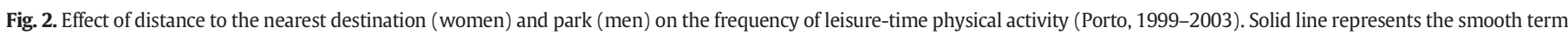
estimating the effect of distances (hm) on the frequency of leisure-time physical activity; gray shade limits the $95 \%$ confidence interval. 
Surprisingly, we found no association between neighborhood SES and LTPA frequency, and no interaction between neighborhood SES and other neighborhood characteristics. Comparisons with other studies are difficult because the role of neighborhood SES on older individuals' PA has been poorly explored and area-level SES measurements vary greatly between studies and settings. Perhaps inevitably then, mixed results are found throughout the literature (King et al., 2005; Michael et al., 2010; Siu et al., 2012; Van Dyck et al., 2010). Furthermore, it is possible that our measurement of area SES did not capture all dimensions of this broad construct, despite being a multidimensional classification.

\section{Limitations}

Nonetheless, study weaknesses need to be acknowledged. Firstly, the cross-sectional design prohibited investigations of causality. Secondly, it relied on self-reported frequency of LTPA. Systematic bias in recollection or reporting remains a possibility. Thirdly, only frequency of PA was measured. There was no discrimination of PA's modalities, only of groups of activities based on energy expenditure. However, the EpiPorto PA Questionnaire was based on a well-established questionnaire (European Prospective Investigation into Cancer and Nutrition) and the validation procedure showed that it is a valid and reproducible instrument for assessing PA among adults (Camões et al., 2010). Fourthly, the study was restricted to a single urban setting with unique characteristics - relatively homogeneous and compact urban design and an equal distribution of socio-demographic characteristics among its inhabitants - which certainly limits the generalizability of the results. Lastly, although we did assess a large number of neighborhood characteristics, we could not include important factors, such as crime, traffic and social support. Likewise, due to data unavailability, we did not incorporate subjective measures of urban environment, which, as seen in previous studies (Inoue et al., 2011; Strath et al., 2012; Van Cauwenberg et al., 2012), might act as important barriers/motivators of PA among older adults and could have contributed to increase the amount of the variability in LTPA explained by our models.

\section{Strengths}

The study has several strengths. The use of objective measures to characterize neighborhood environment reduced the risk of bias associated with subjective measures, frequently seen as a cause of inconsistencies between studies (Koeneman et al., 2011). Our study also captured a wide range of attributes from sport infrastructures to physical environment, maximizing the chance that important correlates were included. Additionally, it was based on a large well-characterized population-based cohort. Being one of the first studies on older population in Southern Europe and the first in Portugal, this represents an important strength, because research should cover diverse regions in order to confirm findings and analyze its generalization potential. Finally, our work fits international and, particularly, European Commission demands in terms of scientific research: Horizon 2020 (framework program for research and innovation) is pushing member states to target their investigation at the field of active aging and age-friendly environments.

\section{Conclusions}

Proximity to shops, cultural sites, places for social interaction and, weakly, parks was associated with increasing time spent on LTPA among the elderly who already participate in PA. However, in this setting, neighborhood characteristics did not define whether older adults were active (some PA) or inactive (no PA at all). From a public health perspective, promoting well distributed destinations and parks could increase the (currently small) percentage of older people who meet PA recommendations. Nevertheless, there is a lack of consensus as to the environmental correlates of PA claims for more longitudinal studies and standardized/validated measures of PA and neighborhood attributes. Given that the pressure over health and social provision systems has been aggravated as demographic aging advances, more attention should be drawn to primary prevention, namely through urban planning interventions.

\section{Conflict of interest statement}

The authors declare that there are no conflicts of interest.

\section{Acknowledgments}

This work was financed by FEDER funds through the Programa Operacional Factores de Competitividade - COMPETE and by Portuguese funds through FCT - Fundação para a Ciência e a Tecnologia in the framework of the project PEst-C/SAU/LA0002/2011 and SFRH/BD/ $82529 / 2011$ fellowship. The authors also acknowledge STCP (Sociedade de Transportes Colectivos do Porto, S.A.) for providing information on public transports. RM was funded by a European Research Council grant [ERC-2010-StG grant 263501].

\section{References}

Baddeley, A., Turner, R., 2013. spatstat: Spatial Point Pattern Analysis, Model-Fitting, Simulation, Tests. R Foundation for Statistical Computing, Vienna, Austria.

Bivand, R., 2013. spdep: Spatial Dependence: Weighting Schemes, Statistics and Models. R Foundation for Statistical Computing, Vienna, Austria.

Camões, M. Severo, M. Santos, A.C. Barros, H. Lopes, C. 2010. Testing an adaptation of the EPIC physical activity questionnaire in Portuguese adults: a validation study that assesses the seasonal bias of self-report. Annals of human biology 37, 185-197.

Cerin, E., Sit, C., Barnett, A., Cheung, M., Chan, W., 2012. Walking for recreation and perceptions of the neighborhood environment in older Chinese urban dwellers. J. Urban Health $90,56-66$.

Chaix, B., Merlo, J., Subramanian, S.V., Lynch, J., Chauvin, P., 2005. Comparison of a spatial perspective with the multilevel analytical approach in neighborhood studies: the case of mental and behavioral disorders due to psychoactive substance use in Malmo, Sweden, 2001. Am. J. Epidemiol. 162, 171-182.

Cohen-Mansfield, J., Marx, M.S., Biddison, J.R., Guralnik, J.M., 2004. Socio-environmental exercise preferences among older adults. Prev. Med. 38, 804-811.

Cunningham, G.O., Michael, Y.L., 2004. Concepts guiding the study of the impact of the built environment on physical activity for older adults: a review of the literature. Am. J. Health. Promot. 18, 435-443.

Dogra, S., Stathokostas, L., 2012. Sedentary behavior and physical activity are independent predictors of successful aging in middle-aged and older adults. J. Aging Health 1-7.

Foster, C., Hillsdon, M., Thorogood, M., 2004. Environmental perceptions and walking in English adults. J. Epidemiol. Community Health 58, 924-928.

Giehl, M.W., Schneider, I.J., Corseuil, H.X., Benedetti, T.R., d'Orsi, E., 2012. Physical activity and environment perception among older adults: a population study in Florianopolis, Brazil. Rev Saude Publ. 46, 516-525.

Gomez, L.F., Parra, D.C., Buchner, D., et al., 2010. Built environment attributes and walking patterns among the elderly population in Bogota. Am. J. Prev. Med. 38, 592-599.

Gremeaux, V., Gayda, M., Lepers, R., Sosner, P., Juneau, M., Nigam, A., 2012. Exercise and longevity. Maturitas.

Hall, K.S., McAuley, E., 2010. Individual, social environmental and physical environmental barriers to achieving 10000 steps per day among older women. Heal. Educ. Res. 25, 478-488.

Hanibuchi, T., Kawachi, I., Nakaya, T., Hirai, H., Kondo, K., 2011. Neighborhood built environment and physical activity of Japanese older adults: results from the Aichi Gerontological Evaluation Study (AGES). BMC Publ. Health 11, 657.

INE, 2001. Resident population (no.) by place of residence (at the date of Census 2001), sex and age group; decennial - Statistics Portugal, population and housing census. Statistics Portugal (INE).

Inoue, S., Ohya, Y., Odagiri, Y., et al., 2011. Perceived neighborhood environment and walking for specific purposes among elderly Japanese. J. Epidemiol. 21, 481-490.

Kavanagh, A.M., Bentley, R., Turrell, G., Broom, D.H., Subramanian, S.V., 2006. Does gender modify associations between self rated health and the social and economic characteristics of local environments? J. Epidemiol. Community Health 60, 490-495.

Kawachi, I., Berkman, L.F., 2003. Neighborhoods and Health. Oxford University Press, New York.

King, W.C., Belle, S.H., Brach, J.S., Simkin-Silverman, L.R., Soska, T., Kriska, A.M., 2005. Objective measures of neighborhood environment and physical activity in older women. Am. J. Prev. Med. 28, 461-469.

Koeneman, M.A., Verheijden, M.W., Chinapaw, M.J., Hopman-Rock, M., 2011. Determinants of physical activity and exercise in healthy older adults: a systematic review. Int. J. Behav. Nutr. Phys. Act. 8, 142

Kremers, S.P., de Bruijn, G.J., Visscher, T.L., et al., 2012. Associations between safety from crime, cycling, and obesity in a Dutch elderly population: results from the Longitudinal Aging Study Amsterdam. J. Environ. Publ. Health. 2012, 127857.

Li, F., Fisher, K.J., Brownson, R.C., Bosworth, M., 2005. Multilevel modelling of built environment characteristics related to neighbourhood walking activity in older adults. J. Epidemiol. Community Health 59, 558-564. 
Li, F., Harmer, P.A., Cardinal, B.J., et al., 2008. Built environment, adiposity, and physical activity in adults aged 50-75. Am. J. Prev. Med. 35, 38-46.

Marin, M., 1988. Gender-differences in sport and movement in Finland. Int. Rev. Sociol. Sport 23, 345-359.

McCormack, G.R., Shiell, A., 2011. In search of causality: a systematic review of the relationship between the built environment and physical activity among adults. Int. J. Behav. Nutr. Phys. Act. 8, 125.

Michael, Y., Beard, T., Choi, D., Farquhar, S., Carlson, N., 2006. Measuring the influence of built neighborhood environments on walking in older adults. J. Aging Phys. Act. 14, 302-312.

Michael, Y.L., Perdue, L.A., Orwoll, E.S., Stefanick, M.L., Marshall, L.M., 2010. Physical activity resources and changes in walking in a cohort of older men. Am. J. Public Health 100 654-660.

Nagel, C.L., Carlson, N.E., Bosworth, M., Michael, Y.L., 2008. The relation between neighborhood built environment and walking activity among older adults. Am. J. Epidemiol. 168, 461-468.

Nathan, A., Pereira, G., Foster, S., Hooper, P., Saarloos, D., Giles-Corti, B., 2012. Access to commercial destinations within the neighbourhood and walking among Australian older adults. Int. J. Behav. Nutr. Phys. Act. 9, 133.

O'Brien, E.A., 2005. Publics and woodlands in England: well-being, local identity, social learning, conflict and management. Forestry 78, 321-336.

Openshaw, S., 1984. The Modifiable Areal Unit Problem. Geo Books, Norwich.

Richardson, E.A., Mitchell, R., 2010. Gender differences in relationships between urban green space and health in the United Kingdom. Soc. Sci. Med. 71, 568-575.

Salvador, E.P., Florindo, A.A., Reis, R.S., Costa, E.F., 2009. Percepção do ambiente e prática de atividade física no lazer entre idosos. Rev Saude Publ. 43, 972-980.

Santos, A.C., Barros, H., 2003. Prevalence and determinants of obesity in an urban sample of Portuguese adults. Public Health 117, 430-437.
Schmand, B., Lindeboom, J., Hooijer, C., Jonker, C., 1995. Relation between education and dementia: the role of test bias revisited. J. Neurol. Neurosurg. Psychiatry 59, 170-174.

Schutzer, K.A., Graves, B.S., 2004. Barriers and motivations to exercise in older adults. Prev. Med. 39, 1056-1061.

Siu, V.W., Lambert, W.E., Fu, R., Hillier, T.A., Bosworth, M., Michael, Y.L., 2012. Built environment and its influences on walking among older women: use of standardized geographic units to define urban forms. J. Environ. Publ. Health. 2012, 1-7.

Stafford, M. Cummins, S, Macintyre, S, Ellaway, A Marmot, M., 2005. Gender differences in the associations between health and neighbourhood environment. Soc. Sci. Med. 60, 1681-1692.

Strath, S.J., Greenwald, M.J., Isaacs, R., et al., 2012. Measured and perceived environmenta characteristics are related to accelerometer defined physical activity in older adults. Int. J. Behav. Nutr. Phys. Act. 9, 40.

Tsunoda, K., Tsuji, T., Kitano, N., et al., 2012. Associations of physical activity with neighborhood environments and transportation modes in older Japanese adults. Prev. Med. 55, 113-118.

Van Cauwenberg, J., Clarys, P., De Bourdeaudhuij, I., et al., 2012. Physical environmenta factors related to walking and cycling in older adults: the Belgian aging studies. BMC Publ. Health 12, 142.

Van Dyck, D., Cardon, G., Deforche, B., Sallis, J.F., Owen, N., De Bourdeaudhuij, I., 2010. Neighborhood SES and walkability are related to physical activity behavior in Belgian adults. Prev. Med. 50 (Suppl. 1), S74-S79.

Van Dyck, D., Cerin, E., Conway, T.L., et al., 2012. Associations between perceived neighborhood environmental attributes and adults' sedentary behavior: findings from the U.S.A., Australia and Belgium. Soc. Sci. Med. 74, 1375-1384.

Warde, A., 2006. Cultural capital and the place of sport. Cult. Trends 15, 107-122.

WHO, 2010. Global Recommendations on Physical Activity for Health.

Wood, S., 2009. mgcv: Mixed GAM Computation Vehicle with GCV/AIC/REML Smoothness Estimation. R Foundation for Statistical Computing, Vienna, Austria. 